\section{(A) Check for updates}

Cite this: Food Funct., 2018, 9, 3185

\title{
Impact of tart cherry juice on systolic blood pressure and low-density lipoprotein cholesterol in older adults: a randomized controlled trial
}

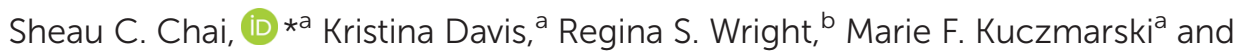 \\ Zugui Zhangc
}

\begin{abstract}
Hypertension and dyslipidemia are major risk factors for cardiovascular disease (CVD). Common treatments for high blood pressure (BP) and dyslipidemia include medications, but there is question as to whether natural sources may be adequate to reduce CVD risk factors. We examined the effects of tart cherry juice on lipid profiles, BP, glucose, insulin, and homeostatic model assessment-insulin resistance (HOMA-IR) in older adults. In this randomized-controlled clinical trial, 17 men and 20 women between the ages of 65-80 years were randomly assigned to consume $480 \mathrm{ml}$ of tart cherry juice or control drink daily for 12 weeks. Control beverages were matched for energy and sugar content. Outcome variables were assessed at baseline and after 12 weeks of tart cherry juice or control drink. Systolic BP and lowdensity lipoprotein cholesterol (LDL) exhibited treatment $\times$ time interaction effects. At the end of the study, participants in the tart cherry group had lower levels of LDL cholesterol (difference of -20.6 with $P=0.001$ ) and total cholesterol (difference of -19.11 with $P=0.01$ ), and higher levels of glucose (difference of 7.94 with $P=0.001$ ), triglycerides (difference of 6.66 with $P=0.01$ ) and BMI (difference of 1.06 with $P=0.02$ ) than in the control group. Neither tart cherry juice nor control significantly altered body weight, high-density lipoprotein cholesterol, diastolic BP, insulin and HOMA-IR. Our findings show that tart cherry juice can lower the levels of systolic BP and LDL cholesterol. However, larger and longer follow-up studies are needed to further assess cardio-protective effects of tart cherry juice.
\end{abstract}

\begin{abstract}
Received 12th March 2018, Accepted 21st May 2018

DOI: $10.1039 / c 8 f 000468 d$

rsc.li/food-function
\end{abstract}

\section{Introduction}

Cardiovascular disease (CVD) continues to be the leading cause of death in the United States. It was estimated that $80 \%$ of Americans who died of CVD were age 65 or older. ${ }^{1}$ Since the number of adults at or above the age of 65 is rapidly increasing and projected to exceed 82 million by the year 2040, CVD can be considered a major health threat. ${ }^{2}$ In addition, the total cost of all types of CVD in 2015 was 656 billion dollars and this figure is expected to nearly double, reaching 1208 billion by the year $2030 .{ }^{1}$ Common treatments for high BP and dyslipidemia include angiotensin-converting enzyme inhibitors, betablockers, calcium antagonists, diuretics, and cholesterol-lowering drugs. Administration of antihypertensive agents for a few years to individuals with high blood pressure (BP) can delay

\footnotetext{
${ }^{a}$ Department of Behavioral Health and Nutrition, College of Health Sciences, University of Delaware, Newark, DE, 19716,USA.E-mail: scchai@udel.edu; Tel: +1 (302) 831-7345

${ }^{b}$ School of Nursing, College of Health Sciences, University of Delaware, Newark, DE, 19716, USA

${ }^{c}$ Value Institute, Christiana Care Health System, Newark, DE, 19718, USA
}

transition to hypertension, but long lasting effects of the intervention and cost-effectiveness remain to be proven. ${ }^{3}$ While these medications have been shown to be effective in the treatment and prevention of CVD, taking these medications regularly may cause negative side effects. Possible side effects include a dry cough, dizziness, bradycardia, peripheral edema and insomnia. These side effects paired with the fact that $84 \%$ of adults over the age of 57 are already taking at least one prescription medication per day, warrant the need for a natural remedy for the problem of CVD. ${ }^{4}$

Consumption of a healthful diet high in fruit and vegetables has been associated with a decreased prevalence of CVD. ${ }^{5,6}$ In addition, consumption of fruits containing various vitamins and polyphenolic compounds may also decrease CVD risk factors. ${ }^{7-9}$ Tart cherries are high in polyphenolic compounds that have antioxidant and anti-inflammatory properties. ${ }^{10-14}$ Health benefits associated with the consumption of tart cherries may include decreased muscle damage following strenuous exercise, improved sleep and circulating melatonin concentrations. ${ }^{15-18}$ There is also emerging evidence that tart cherries exert cardioprotective effects in animal models. ${ }^{19,20}$ So far there is little evidence on 
humans. ${ }^{21,22}$ Based on the results of previous studies, tart cherries and their bioactive components have been shown to improve cardiovascular performance. ${ }^{19-22}$ Despite this evidence, the ability of tart cherries to impact cardiovascular risk factors has not yet been investigated in older adults. Therefore, the present study was conducted to assess the ability of tart cherry juice consumption to reduce CVD risk factors by assessing lipid profiles, BP, glucose, insulin and homeostatic model assessment-insulin resistance (HOMA-IR) in older adults.

\section{Methods}

\subsection{Study design and participants}

The present study was a 12-week, parallel, randomized controlled trial conducted at the University of Delaware in the United States. Men and women of diverse race and ethnicities who live in Newark, Delaware and the surrounding areas were recruited. Recruiting materials were placed in the local community, including senior living facilities, event halls, churches, and public bulletin boards. Advertisements were also placed in the Delaware News Journal. A total of 284 men and women were screened for eligibility. Following the initial phone screening, 129 potential participants were invited to the study site to complete a screening visit. A medical history, medication/supplement use, food allergies, and typical food intake were obtained from participants for screening purposes. Inclusion criteria included men and women between the ages of 65-80 years who consumed $\leq 5$ servings of fruits and vegetables per day. Individuals receiving treatment with any medications that may influence brain function or have had any prior diagnosis or history of stroke, heart disease, diabetes, gastrointestinal disease, cancer, central nervous system or psychiatric disorders, traumatic brain injury, or impaired cognitive function were excluded. Additionally, heavy smokers ( $>20$ cigarettes per day) or individuals allergic to tart cherry were excluded from this study. Based on inclusion and exclusion criteria, a total of 37 participants were enrolled in the study and randomized to intervention. Eligible men and women were randomly assigned to consume either $480 \mathrm{ml}$ tart cherry juice or control drink daily for 12 weeks by a researcher using a simple randomization and a pre-generated randomization list generated by a computer program. Participants were blinded to which group they were in until the study was closed. Researchers and personnel, including the biostatistician, were blinded to the groups when measuring BP and anthropometrics, running the assays, and analyzing the data. This study was conducted according to the guidelines laid down in the Declaration of Helsinki and all procedures involving human subjects were approved by the Institutional Review Board at the University of Delaware. All participants provided written informed consent before participating in the study. The trial was registered at clinicaltrials.gov as NCT02922920.

\subsection{Intervention}

A commercially available Montmorency tart cherry concentrate (King Orchards, MI) was used in this study. The nutrient compositions of the tart cherry concentrate are presented in Table 1. The control drink was isocaloric, devoid of tart cherry or its polyphenols, with similar color, sugar content, acid, and flavor as the tart cherry juice (Table 1). To prepare $480 \mathrm{ml}$ tart cherry juice, $68 \mathrm{ml}$ Montmorency tart cherry juice concentrate (approximately $514 \mathrm{~g}$ of whole tart cherries) was diluted with $412 \mathrm{ml}$ water. Control drink was prepared by mixing unsweetened black cherry flavored Kool-Aid (Kraft Foods, United States) with water. Dextrose and fructose were added to match the carbohydrate content found in tart cherry. Additional food dye and lemon flavored powder were added to match the color and tartness of the tart cherry juice. All beverages were prepared specifically according to the instructions and properly stored in freezer/refrigerator until consumption. Participants were given their assigned beverage and instructions regarding consumption and storage. Participants were instructed to consume $480 \mathrm{ml}$ of the beverage per day, $240 \mathrm{ml}$ in the morning and $240 \mathrm{ml}$ in the evening for the entirety of the 12-week intervention. Participants were asked to pick up their fresh supplements on a monthly basis. In addition, participants were given customized calendars and were asked to mark the days they missed consuming the study regimen and to return any unused portion for compliance monitoring purposes.

\subsection{Blood pressure and anthropometric measurements}

Resting BP and anthropometric measurements were collected at baseline and after the 12-week dietary intervention. A digital BP monitor (HEM-907XL, Omron Healthcare, Inc.) was used for measuring BP. Two measurements were taken after participants sat in a quiet room with their feet placed on the ground for five minutes. Results were recorded as the average of the two measurements. A stadiometer was used to measure height to the nearest $0.1 \mathrm{~cm}$. Participants stood barefoot with their backs against the wall and their heads in the Frankfort hori-

Table 1 Nutrient composition of tart cherry and control beverages

\begin{tabular}{lll}
\hline & $\begin{array}{l}\text { Tart cherry } \\
(480 \mathrm{ml})\end{array}$ & $\begin{array}{l}\text { Control } \\
(480 \mathrm{ml})\end{array}$ \\
\hline Energy (kcal) & 181 & 180 \\
Carbohydrate (g) & 43 & 45 \\
Dietary fiber (g) & - & - \\
Protein (g) & 2.3 & - \\
Fat (g) & - & - \\
Calcium (mg) & 8.6 & - \\
Phosphorous (mg) & 46 & - \\
Potassium (mg) & 355 & - \\
Thiamin (mcg) & 11 & - \\
Folic acid (mcg) & 18.6 & - \\
ORAC (unit) & 6092.8 & \\
Total tannins (mg) & 95.9 & - \\
Total phenolics (gallic acid equivalents) & 450.6 & - \\
ORAC - oxygen radical absorption capacity. & & -
\end{tabular}


zontal plane. Weight was measured in kilograms with minimal clothing on a digital scale to the nearest $\pm 0.1 \mathrm{~kg}$. Body mass index (BMI, $\mathrm{kg} \mathrm{m}^{-2}$ ) was calculated from the height and weight measurements.

\subsection{Serum lipid profile, and glucose assessments}

Fasting venous blood for plasma and serum was collected after an overnight fast at baseline and after the 12-week intervention. Serum and plasma were separated by centrifuging at $1500 \mathrm{~g}$ for 15 minutes at $4^{\circ} \mathrm{C}$ using a Beckman Allegra 6KR centrifuge (Beckman Coulter, Inc.). Samples were then aliquoted and stored at $-80{ }^{\circ} \mathrm{C}$ until analyses. Serum was analyzed for total cholesterol (TC; inter-assay CV: 1.1\%; intra-assay CV: $0.8 \%$ ), high-density lipoprotein cholesterol (HDL; inter-assay CV: $4.7 \%$; intra-assay CV: $5.1 \%$ ), triglyceride (TG; inter-assay CV: $1.90 \%$; intra-assay CV: $1.82 \%$ ) and glucose in duplicate at baseline and after 12-week intervention using a Genesys spectrophotometer (Thermo Scientific, Waltham, MA). All reagents and standards were from Pointe Scientific, Inc. After determining serum TC, HDL, and TG levels, serum low-density lipoprotein cholesterol (LDL) level was calculated using the Friedewald equation. ${ }^{23}$ Serum insulin were measured using ELISA kit from EMD Millipore Corporation (inter-assay CV: 6.6\%; intra-assay CV: $1.8 \%$; Saint Charles, MO, USA). HOMA-IR was calculated as fasting glucose $\left(\mathrm{mg} \mathrm{dL}^{-1}\right)$ multiplied by fasting insulin $\left(\mu \mathrm{U} \mathrm{mL}^{-1}\right)$ divided by 405 .

\subsection{Dietary and physical activity assessments}

Study participants were asked to maintain their normal dietary habit and physical activity throughout the study. To monitor dietary intake and physical activity, a 3-day food record (two weekdays and one weekend day) and the Physical Activity Scale for the Elderly questionnaire (PASE) ${ }^{24}$ were assessed at baseline and 12-week dietary intervention. Collected food records were analyzed using Nutrition Data System for Research software (NDSR, Minneapolis, MN).

\subsection{Statistical analyses}

All analyses were performed using SAS 9.3 (SAS Institute Inc., Cary, NC). Descriptive statistics were used to describe all data. Variables were summarized using means and standard deviations for continuous data, and percentages and frequencies for categorical data. Comparisons for baseline sociodemographic characteristics, including sex and age, were performed. The Wilcoxon rank sum test was used to compare continuous variables between two groups, including the mean changes in outcome variables during the intervention period, and contingency table analysis (chi-square) was used to compare categorical variables. In comparisons, differences with $P<0.05$ were considered significant. The mean change from baseline to the end of the intervention was computed in each of the two groups. The effect of tart cherry juice on final outcome variables was performed via ANCOVA analysis, with adjustment for physical activity and dietary cholesterol, which showed significant baseline differences between tart cherry and control group. The final adjusted outcome variables based on the
ANCOVA were compared, and utilized to examine the impact of treatment, time, and the interaction of treatment and time via mixed ANOVA. Significant $P$ values from the mixed ANOVA were reported for treatment effect, time effect, and interaction of treatment and time.

\section{Results}

\subsection{Attrition rate and compliance}

A flow chart of participants is presented in Fig. 1. Of the 37 persons who participated in this 12 -week, randomized controlled trial, 34 participants (94.2\% compliance) successfully completed. Reasons for withdrawal included noncompliance with the study protocol, loss of interest, and an unrelated medical condition. Tart cherry juice and control drink were well tolerated by the participants. No adverse events were reported in the study.

\subsection{Baseline characteristics, anthropometric measurements, dietary intake and physical activity}

Participant characteristics and demographic characteristics of participants are presented in Table 2. There were no significant differences between these characteristics except for income and employment status. Mean ( \pm standard deviation) age was $69.5 \pm 3.9$ and $70.0 \pm 3.7$ years, in the control and tart cherry groups, respectively. The mean BMI indicated overweight status. The majority of the participants had at least a 4-year college degree and were White and married. In the tart cherry group, half of the participants were working while half were retired; in the control group, the majority of participants were retired.

The baseline mean physical activity score was higher and the baseline mean dietary cholesterol was significantly lower in the tart cherry group than in the control group, respectively. As a result, centered baseline physical activity score and dietary cholesterol and the terms of their interaction with the intervention variable were included in the ANCOVA model. The results showed that the final BMIs were significantly different between the two groups (28.6 in tart cherry vs. 27.5 in control

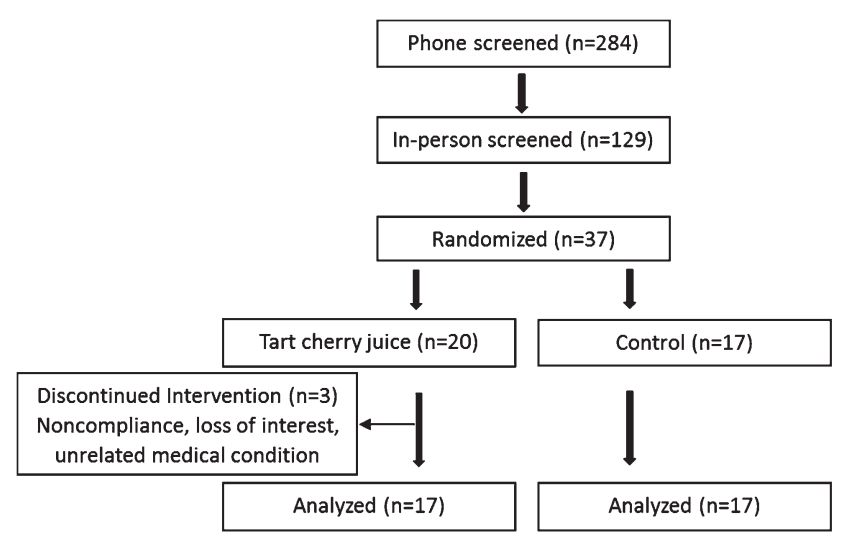

Fig. 1 Flow of the study participants. 
Table 2 Baseline characteristics of study participants

\begin{tabular}{|c|c|c|c|}
\hline Variables & $\begin{array}{l}\text { Control } \\
(n=17)\end{array}$ & $\begin{array}{l}\text { Tart cherry } \\
(n=20)\end{array}$ & $P$-Value \\
\hline & Mean $\pm \mathrm{SD}$ & Mean $\pm \mathrm{SD}$ & \\
\hline Age, years & $69.5 \pm 3.9$ & $70.0 \pm 3.7$ & 0.65 \\
\hline Body height, cm & $169.6 \pm 7.9$ & $165.5 \pm 6.8$ & 0.25 \\
\hline Body mass, kg & $78.7 \pm 13.5$ & $78.0 \pm 10.3$ & 0.56 \\
\hline \multirow[t]{2}{*}{$\mathrm{BMI}, \mathrm{kg} \mathrm{\textrm {cm } ^ { - 2 }}$} & $27.3 \pm 4.2$ & $28.5 \pm 3.7$ & 0.34 \\
\hline & $n(\%)$ & $n(\%)$ & \\
\hline Sex, male/female & $9 / 8(53 / 47)$ & $8 / 12(40 / 60)$ & 0.43 \\
\hline Education Level & & & 0.63 \\
\hline Completed high school & $5(29)$ & $5(25)$ & \\
\hline 2-year college degree & $1(6)$ & $4(20)$ & \\
\hline 4-year college degree & $6(36)$ & $7(35)$ & \\
\hline Higher education & $5(29)$ & $4(20)$ & \\
\hline Income & & & 0.05 \\
\hline Under 25000 & $0(0)$ & $3(15)$ & \\
\hline $25000-49999$ & $1(6)$ & $0(0)$ & \\
\hline $50000-74999$ & $2(12)$ & $8(40)$ & \\
\hline 75 000-99999 & $3(18)$ & $0(0)$ & \\
\hline Over 100000 & $8(46)$ & $5(25)$ & \\
\hline Prefer not to say & $3(18)$ & $4(20)$ & \\
\hline Race & & & 0.37 \\
\hline Asian & $0(0)$ & $2(10)$ & \\
\hline Black or African American & $2(12)$ & $1(5)$ & \\
\hline White & $15(88)$ & $16(80)$ & \\
\hline Prefer not to say & $0(0)$ & $1(5)$ & \\
\hline Marital status & & & 0.87 \\
\hline Single never married & $1(6)$ & $2(10)$ & \\
\hline Separated/divorced & $3(18)$ & $4(20)$ & \\
\hline Married & $13(76)$ & $14(70)$ & \\
\hline Employment status & & & 0.01 \\
\hline Retired & $15(88)$ & $10(50)$ & \\
\hline Working & $2(12)$ & $10(50)$ & \\
\hline
\end{tabular}

Values are presented as means \pm standard deviation (SD) for continuous variables, and numbers (percentages) for categorical variables. BMI - body mass index.

group; mean difference of 1.06 ; 95\% CI: 0.18 to $1.94 ; P=0.02$ ), but without significant impacts of time, treatment, and interaction of time and treatment (Table 3). Similar results were shown for the total fiber intake (Table 4). By the end of the study, the final dietary fiber intake was significantly lower in the tart cherry group compared with the control group (16.5 g in tart cherry vs. $20.0 \mathrm{~g}$ in control group; mean difference of -3.50 ; 95\% CI: -6.49 to $-0.52 ; P=0.03$ ).

The within group analysis showed that physical activity level significantly decreased in the tart cherry group $(P<0.0001)$ and control group $(P<0.0001)$. The final physical activity levels were significantly higher in the tart cherry group compared with the control group (mean difference of 64.4 ; 95\% CI: 23.7 to 105.0; $P=0.01)$. The main effect of time $(P=0.0003)$ and treatment $(P=0.02)$ were found, but there was no significant interaction by treatment and time $(P=0.23)$. Dietary cholesterol significantly increased in the tart cherry group $(P<0.001)$ and control group $(P=0.04)$. The final dietary cholesterol was significantly lower in the tart cherry group compared with the control group (mean difference of -39.5 ; 95\% CI: -73.48 to $-5.53 ; P=0.03$ ). The main effect of time $(P=0.02)$ and treatment $(P=0.0003)$ were also found, but there was no significant interaction by treatment and time $(P=0.73)$.

\subsection{Systolic and diastolic blood pressures}

Baseline levels of systolic and diastolic BP were not statistically different between tart cherry and control groups (Table 3). There was a significant interaction between treatment and time $(P=0.04)$ for systolic BP. In the baseline, participants in the tart cherry group (141.4 $\mathrm{mmHg}$ ) had higher systolic BP than the control group (133.4 $\mathrm{mmHg}$ ). After the 12 weeks intervention, participants in the tart cherry group had lower systolic BP than the control group, indicating the impact of tart cherry (changes of $-4.1 \mathrm{mmHg}$ in tart cherry vs. changes of $+5.4 \mathrm{mmHg}$ in control, $P=0.04$ ). Diastolic BP remained relatively unchanged in both groups. There were no treatment, time, or treatment $\times$ time interaction effects observed for diastolic BP.

\subsection{Lipid profiles}

Baseline levels of TC, HDL, LDL, and TG were not statistically different between tart cherry and control groups (Table 3). There was a significant interaction between treatment and time $(P=0.02)$ for LDL. After the 12 weeks intervention, participants in the tart cherry group had lower LDL than the control group. The difference was significant (difference of -20.6 ; $95 \%$ CI: -30.67 to -10.6 ; $P=0.001)$.

There were no treatment, time, or treatment $\times$ time interaction effects observed for TC, HDL, and TG. Of note, the final TC was significantly different between two groups (153.8 $\mathrm{mg} \mathrm{dL}^{-1}$ in tart cherry vs. $172.9 \mathrm{mg} \mathrm{dL}^{-1}$ in control group; mean difference of -19.11 ; $95 \%$ CI: -30.00 to $-8.21 ; P=0.01$ ). Similarly, the final TG between two groups was significantly different (mean difference of 6.66; 95\% CI: 2.07 to 11.27; $P=0.01)$.

\subsection{Glucose, insulin and HOMA-IR}

Baseline levels of glucose, insulin and HOMA-IR were not statistically different between tart cherry and control groups (Table 3). There were no treatment, time, or treatment $\times$ time interaction effects observed for insulin and HOMA-IR. Twelve weeks supplementation with tart cherry juice or control drink did not affect levels of insulin and HOMA-IR. The final glucose levels in the tart cherry group were significantly higher than in the control group (mean difference of $7.94 ; 95 \%$ CI: 3.50 to 12.38; $P=0.001)$.

\section{Discussion}

There are a number of studies demonstrating that diets high in fruit and vegetables improve overall cardiovascular health. ${ }^{5-9}$ Our findings indicate that there was a significant impact of tart cherry juice on systolic BP. After the 12 weeks intervention, participants in the tart cherry group had lower systolic BP than the control group, with a decrease in systolic $\mathrm{BP}$ in the tart cherry group and an increase in systolic BP in the control group. Previous studies have found similar effects on BP by consuming tart cherry concentrate, but they employed different study designs, populations, and dosages of 
Table 3 Anthropometric, blood pressure, glucose, and lipid profiles in older adults at baseline and 12 weeks after supplementation with tart cherry juice or control drink

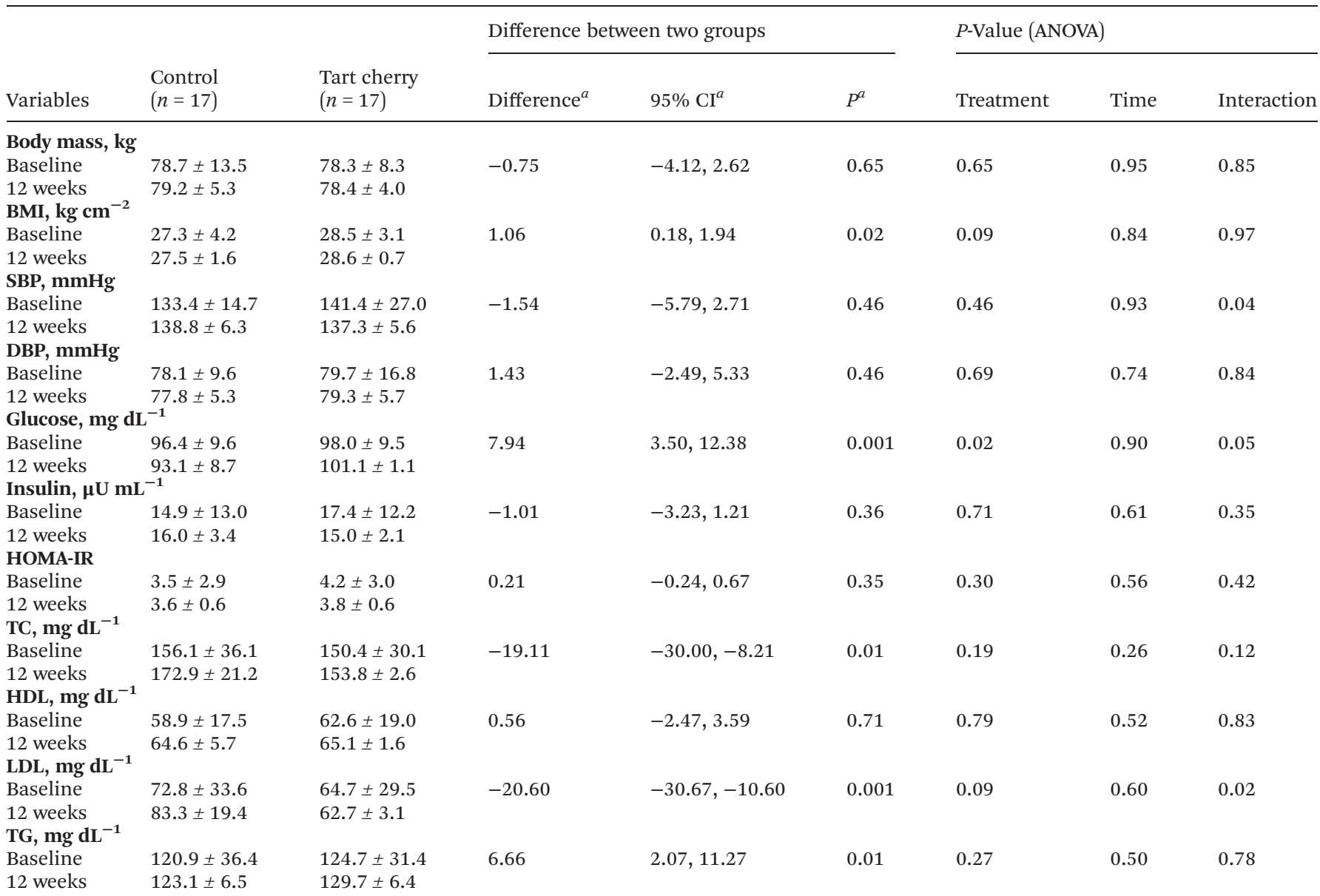

Values are means \pm standard deviation (SD). BMI - body mass index; DBP - diastolic blood pressure; HDL - high-density lipoprotein cholesterol; LDL - low-density lipoprotein cholesterol; HOMA-IR - insulin resistance index; SBP - systolic blood pressure; TC - total cholesterol; TG triglyceride. ${ }^{a}$ Values are adjusted for baseline physical activity and dietary cholesterol, assessed by ANCOVA.

tart cherry concentrate. For instance, Ataie-Jafari et $a l^{21}$ demonstrated that consumption of $40 \mathrm{~g}$ concentrated tart cherry juice for six weeks significantly reduced both systolic and diastolic BP in individuals with type 2 diabetes. Keane et $a l .^{22}$ demonstrated that $60 \mathrm{ml}$ Montmorency tart cherry juice concentrate acutely lowered systolic BP over a period of three hours in younger men with early hypertension compared to control group. However, Lynn et al. ${ }^{25}$ conducted a 6-week open label trial where healthy participants between the ages of 30-50 consumed $30 \mathrm{ml}$ of a Montmorency tart cherry concentrate or a control beverage. They found that six weeks consumption of $30 \mathrm{ml}$ tart cherry concentrate daily had no effects on arterial stiffness and BP. It should be noted that this intervention trial was only 6 weeks and there may need to be a longer intervention period and a higher dose of tart cherry concentrate to notice significant changes especially in arterial stiffness.

Tart cherries are a rich source of polyphenolic compounds, especially proanthocyanins, anthocyanins, and flavonols, all of which are strong antioxidants. ${ }^{10}$ Tart cherries are a particularly rich source of anthocyanins, specifically cyanidin-3-glucosylrutinoside, cyanidin-3-rutinoside, cyanindin-3-glucoside, and their agylcone, cyaniding. ${ }^{26,27}$ It has been proposed that the mechanism behind tart cherry's health benefits is related to the bioactive compounds present in tart cherries, including various polyphenolic compounds that act as antioxidants. Thus, it can be reasoned that the reduction in systolic BP seen in our present study was due in part to the presence of polyphenolic compounds in the tart cherry juice. Diebolt et al. ${ }^{28}$ reported a significant reduction in systolic BP following a short-term oral administration of polyphenolic compounds in rats. Changes in $\mathrm{BP}$ could be related to observed changes in vascular reactivity of the thoracic aorta leading to enhanced endothelium relaxation and an induction of gene expression (of inducible NO synthase and cyclooxygenase-2), therefore leading to reduced BP. Another study showed that systolic and diastolic BPs were significantly reduced in adults following eight weeks consumption of drinks containing approximately 
Table 4 Dietary intake and physical activity in older adults at baseline and 12 weeks after supplementation with tart cherry juice or control drink

\begin{tabular}{|c|c|c|c|c|c|c|c|c|}
\hline \multirow[b]{2}{*}{ Variables } & \multirow[b]{2}{*}{ Control $(n=17)$} & \multirow[b]{2}{*}{ Tart cherry $(n=17)$} & \multicolumn{3}{|c|}{ Difference between two groups } & \multicolumn{3}{|c|}{$P$-Value (ANOVA) } \\
\hline & & & Difference $^{a}$ & $95 \% \mathrm{CI}^{a}$ & $P^{a}$ & Treatment & Time & Interaction \\
\hline \multicolumn{9}{|c|}{ Energy, kcal } \\
\hline Baseline & $2009 \pm 526$ & $1709 \pm 610$ & -162.5 & $-356.6,61.57$ & 0.09 & 0.04 & 0.27 & 0.80 \\
\hline 12 weeks & $1880 \pm 114$ & $1717 \pm 375$ & & & & & & \\
\hline \multicolumn{9}{|c|}{ Carbohydrate, $\mathrm{g}$} \\
\hline $\begin{array}{l}\text { Baseline } \\
12 \text { weeks }\end{array}$ & $\begin{array}{l}234.1 \pm 71.8 \\
221.3 \pm 13.2\end{array}$ & $\begin{array}{l}196.0 \pm 86.7 \\
181.8 \pm 56.9\end{array}$ & -39.5 & $-68.3,-10.56$ & 0.02 & 0.02 & 0.15 & 0.72 \\
\hline \multicolumn{9}{|l|}{ Protein, $g$} \\
\hline $\begin{array}{l}\text { Baseline } \\
12 \text { weeks }\end{array}$ & $\begin{array}{l}86.3 \pm 30.5 \\
75.1 \pm 7.2\end{array}$ & $\begin{array}{l}75.6 \pm 27.3 \\
74.1 \pm 22.1\end{array}$ & -0.92 & $-12.45,10.61$ & 0.87 & 0.18 & 0.13 & 0.47 \\
\hline \multicolumn{9}{|l|}{ Fat, $\mathrm{g}$} \\
\hline $\begin{array}{l}\text { Baseline } \\
12 \text { weeks }\end{array}$ & $\begin{array}{l}75.0 \pm 19.1 \\
76.2 \pm 4.0\end{array}$ & $\begin{array}{l}67.4 \pm 29.4 \\
75.6 \pm 10.3\end{array}$ & -0.55 & $-6.05,4.95$ & 0.84 & 0.35 & 0.55 & 0.74 \\
\hline \multicolumn{9}{|c|}{ Total fiber, $\mathrm{g}$} \\
\hline $\begin{array}{l}\text { Baseline } \\
12 \text { weeks }\end{array}$ & $\begin{array}{l}21.4 \pm 7.9 \\
20.0 \pm 3.4\end{array}$ & $\begin{array}{l}20.7 \pm 12.0 \\
16.5 \pm 4.9\end{array}$ & -3.5 & $-6.49,-0.52$ & 0.03 & 0.11 & 0.07 & 0.34 \\
\hline \multicolumn{9}{|c|}{ Dietary cholesterol, mg } \\
\hline $\begin{array}{l}\text { Baseline } \\
12 \text { weeks } \\
\text { PASE }\end{array}$ & $\begin{array}{l}262.0 \pm 133.9 \\
333.9 \pm 14.6 \dagger\end{array}$ & $\begin{array}{l}203.0 \pm 90.3 \\
294.4 \pm 67.1 \S\end{array}$ & -39.5 & $-73.48,-5.53$ & 0.03 & 0.02 & 0.0003 & 0.73 \\
\hline $\begin{array}{l}\text { Baseline } \\
12 \text { weeks }\end{array}$ & $\begin{array}{l}135.8 \pm 37.3 \\
126.3 \pm 31.3 \ddagger\end{array}$ & $\begin{array}{l}224.5 \pm 90.1 \\
190.6 \pm 75.6 \$\end{array}$ & 64.4 & $23.7,105.0$ & 0.01 & 0.0003 & 0.02 & 0.23 \\
\hline
\end{tabular}

Values are means \pm standard deviation (SD). PASE - physical activity scale for the elderly. ${ }^{a}$ Values are adjusted for baseline physical activity and dietary cholesterol, assessed by ANCOVA. $\dagger P<0.05$ for within-group differences in comparison with baseline. $\S P<0.001$ for within-group differences in comparison with baseline. $\ddagger P<0.0001$ for within-group differences in comparison with baseline.

$837 \mathrm{mg}$ of polyphenols. ${ }^{29}$ This reduction could be attributable to the action of polyphenolic compounds as determined by bioavailability analysis. The proper functioning of vascular smooth muscle cell (VSMC) is essential to avoid build up of atherosclerotic plaques, as VSMCs are responsible for normal, healthy vasculature tone. The decrease in systolic BP may be associated with plasma protocatechuic acid and vanillic acid as these anthocyanin metabolite have been shown to modulate VSMCs in vitro. ${ }^{30-32}$ Other juices, such as beetroot and blueberry, have also shown cardiovascular benefits, including reduced BP. Hobbs et al. ${ }^{33}$ demonstrated that 90 minutes after ingestion of $100 \mathrm{~g}$ beetroot juice, systolic and diastolic BPs began to decrease. The peak reductions in systolic and diastolic BPs, occurring at 2-3 hours, were $13.1 \mathrm{mmHg}$ and 16.6 mmHg, respectively. Beetroot juice contains high amounts of betalains and inorganic nitrate. Following consumption of the beetroot juice, urinary nitric oxide, a potent vasodilator, was significantly increased resulting in a reduction in systolic and diastolic BPs. Johnson et al. ${ }^{8}$ also demonstrated that 8 weeks consumption of drinks containing $22 \mathrm{~g}$ freezedried blueberry significantly decreased systolic and diastolic BPs by $5.1 \%$ and $6.3 \%$, respectively, in postmenopausal women. The authors concluded that the reduction of BP may be due, in part, to increased nitric oxide production. Blueberries, similarly to tart cherries, have a high anthocyanin content and this may have played a role in the hypotensive effects noted following blueberry supplementation.

Another component of tart cherry that may influence its effects on BP is its potassium content. Adequate dietary potassium is important for BP and heart health. Various studies have shown that across a wide range of baseline intakes (ranging from less than 50 to greater than $80 \mathrm{mmol}$ ), increasing potassium intake provides beneficial in reducing systolic BP by $3.39-4.11 \mathrm{mmHg} .{ }^{34-36}$ A population study conducted by Khaw et al. ${ }^{37}$ also suggested that an increase in potassium by 20-30 mmol per day ( 700-1200 mg per day), which resulted in a 2 to $3 \mathrm{mmHg}$ reduction in systolic BP. A meta-analysis that included 33 studies concluded that potassium supplementation significantly reduced systolic BP by $3.11 \mathrm{mmHg}$ and diastolic BP by $1.97 \mathrm{mmHg} .{ }^{34}$ A diet rich in potassium such as the Dietary Approaches to Stop Hypertension diet has been shown to be effective in reducing systolic and diastolic BP by 11.4 and $5.5 \mathrm{mmHg}$, respectively, in hypertensive individuals. ${ }^{38}$ Another meta-analysis found that an increased potassium intake of $90-120 \mathrm{mmol}$ day $^{-1}$ resulted in a reduction in systolic and diastolic BP of $7.16 \mathrm{mmHg}$ and $4.01 \mathrm{mmHg}$, respectively. ${ }^{39}$ The estimated potassium content of the tart cherry supplement provided in this study was approximately $355 \mathrm{mg}$. It is unlikely that the tart cherry concentrate used in the present study provided potassium in high enough quantities to elicit significant improvement in BP. Tart cherries contain various vitamins, minerals and polyphenolic compounds. The exact nutrients and/or components contributing to the BP-lowering effects of tart cherry are unknown. However, many of these compounds are known to exert cardioprotective effects and therefore likely work additively and/or synergistically. Our finding provides the evidence that tart cherry juice consumption was able to reduce systolic BP by $4.1 \mathrm{mmHg}$, from $141.4 \mathrm{mmHg}$ to $137.3 \mathrm{mmHg}$ in older adults. This reduction of systolic BP is quite promising. As reported in 
the Framingham Heart study, individuals with $\mathrm{BP} \geq 140 / 90$ were 1.5 times more likely to have CVD and 1.6 times more likely to die from CVD complications when compared to individuals with $\mathrm{BP}<140 / 90 \mathrm{mmHg} .{ }^{40}$ In addition, another study demonstrated that a $2 \mathrm{mmHg}$ reduction in $\mathrm{BP}$ can reduce stroke mortality by $6 \%$ and coronary heart disease mortality by $4 \%{ }^{41}$ Therefore, this shift of BP in our study is important in improving overall cardiovascular health, which in turn improves longevity and quality of life.

Tart cherry concentrate is relatively high in natural sugar compared to many other fruits. With respect to the influence of tart cherry on lipid profiles, glucose and insulin sensitivity, our study has demonstrated that there was a significant impact of tart cherry juice on LDL cholesterol. Evidence suggests that increased levels of LDL cholesterol is associated with increased risks of CVD events and atherosclerosis. ${ }^{42,43}$ After the 12 weeks intervention, participants in the tart cherry group had lower LDL than the control group, with a decrease in LDL in the tart cherry group and an increase in LDL in the control group. Twelve weeks supplementation with tart cherry juice did not affect the levels of insulin and HOMA-IR. However, the final glucose levels in tart cherry group were higher in control group. A possible mechanism associated with the reduction in LDL cholesterol following supplementation with tart cherry may due to the ability of tart cherry to bind bile acids. Research has shown that cherries can bind bile acids up to $5 \% .{ }^{44}$ Another study showed that a cherry extract containing $20 \mu \mathrm{M}$ per GAE of phenolic compounds completely inhibited LDL oxidation. ${ }^{45}$ Both of these studies used sweet cherries. As it has been shown that tart cherries contain higher levels of polyphenolic compounds, it can be expected that tart cherries may lead to similar, if not even more significant results. It is important to note the present study population is generally healthy older adults. The cholesterol-lowering effects of tart cherry juice may be more effective on obese individuals or individuals with dyslipidemia.

Our present study also demonstrated that there was a significant impact of control drink on systolic BP and LDL cholesterol. Systolic BP and LDL cholesterol nonsignificantly increased after 12 weeks of control drink consumption when compared with baseline values. The sugar content of the control drink, which contained fructose may, in part, be responsible for the increase in LDL cholesterol and systolic BP. Jameel et $a l^{46}$ demonstrated that 30 and 60 minutes after ingestion of $50 \mathrm{~g}$ fructose, both LDL and C-reactive protein were elevated in healthy individuals. Brown et $a .^{47}$ also demonstrated that 60 minutes after ingestion of $60 \mathrm{~g}$ fructose, $\mathrm{BP}$, heart rate, cardiac output, and BP variability all increased, and cardiovagal baroreflex sensitivity decreased, in young adults. In a study by Litterio et al. ${ }^{48}$ they found that rat fed chow diet supplemented with $10 \%$ (w/v) fructose in the drinking water for 4 weeks evidenced significantly increased systolic BP. The level of systolic BP was further increased after 8 weeks of high fructose diet. A study conducted by Stanhope et al. ${ }^{49}$ also suggested that 8 weeks consumption of fructose swee- tened beverage at $25 \%$ of energy requirements significantly increased dyslipidemia and decreased insulin sensitivity without significantly altering BP and glucose.

Our findings suggest that daily incorporation of tart cherry juice or control ( $\sim 181 \mathrm{kcal}$ per day) into a diet, without intentional dietary modification during the study, do not significantly affect the total energy intake of older adults. According to NHANES data, adults are consuming approximately $209 \mathrm{kcal}$ per day from sugar-sweetened beverages. ${ }^{50}$ Our 3-day food records show that the majority of the participants replaced their average daily sugar-sweetened drink with the tart cherry juice or control drink. In addition, some participants have altered their dietary intake to compensate for the tart cherry juice or control drink. Therefore, there was no overall increase in energy intake and no significant increase in weight.

The strengths of this present study include the randomized controlled trial study design. Participants were blinded to which group they were in and juices were similar in color and taste. In addition, the analysis of possible confounding variables including dietary intake and physical activity were assessed. This study is not without limitations. Study results could be influenced by various factors such as sample size, the duration of the tart cherry supplementation, and the possibility of under or over reporting dietary intake or differences in physical activity at baseline between groups. In the present study, participants were asked to maintain their habitual diet and physical activity levels. However, the physical activity level significantly decreased and dietary cholesterol significantly increased in the tart cherry group and control group, respectively, indicating poor compliance with the instruction. It is important to recognize that physical activity and dietary intake vary between seasons, and the physical activity and three-day food records were able to take that into consideration. All food records were reviewed with participants for completion and accuracy. In order to eliminate the effects of confounding factors including physical activity and dietary cholesterol, the ANCOVA test was used. A total of three participants withdrew from the intervention, therefore reducing the power to detect changes. Finally, the majority of the research sample was White, married and earned $\geq \$ 75000$ per year which limits the generalizability of findings.

\section{Conclusion}

In conclusion, daily incorporation of tart cherry juice into the diet can lower systolic BP and LDL cholesterol in older adults and could be a plausible intervention for improved cardiovascular health for this population. Our findings warrant further investigation in a larger sample size and longer-term trials.

\section{Author contributions}

The authors' responsibilities were as follows: S. C. C. designed the study and conducted the research, interpreted the data, 
wrote the manuscript, and had primary responsibility for the final content of the manuscript; K. D. conducted the research and wrote the manuscript; R. S. W. and M. F. K. wrote the manuscript; Z. Z., a biostatistician performed the statistical analysis and interpreted the data; and all authors reviewed and approved the final manuscript.

\section{Conflicts of interest}

The authors declare that there are no conflicts of interest.

\section{Acknowledgements}

The present study was supported by the Cherry Research Committee of the Cherry Marketing Institute, a non-profit organization. Tart cherry concentrates were provided by the Cherry Marketing Institute. Funders had no role in the study design, data collection, data analysis or interpretation, or writing of the manuscript.

\section{References}

1 D. Mozaffarian, E. J. Benjamin, A. S. Go, D. K. Arnett, M. J. Blaha, M. Cushman, S. de Ferranti, J. P. Despres, H. J. Fullerton, V. J. Howard, M. D. Huffman, S. E. Judd, B. M. Kissela, D. T. Lackland, J. H. Lichtman, L. D. Lisabeth, S. Liu, R. H. Mackey, D. B. Matchar, D. K. McGuire, E. R. Mohler 3rd, C. S. Moy, P. Muntner, M. E. Mussolino, K. Nasir, R. W. Neumar, G. Nichol, L. Palaniappan, D. K. Pandey, M. J. Reeves, C. J. Rodriguez, P. D. Sorlie, J. Stein, A. Towfighi, T. N. Turan, S. S. Virani, J. Z. Willey, D. Woo, R. W. Yeh, M. B. Turner, C. American Heart Association Statistics and S. Stroke Statistics, Heart disease and stroke statistics-2015 update: a report from the American Heart Association, Circulation, 2015, 131, e29322.

2 U. S. Census Bureau, Projections of the size and composition of the U.S. population: 2014 to 2060, https://census. gov/content/dam/Census/library/publications/2015/demo/p251143.pdf, (accessed 3/1/2018, 2018).

3 M. Banach, C. Serban, W. S. Aronow, J. Rysz, S. Dragan, E. V. Lerma, M. Apetrii and A. Covic, Lipid, blood pressure and kidney update 2013, Int. Urol. Nephrol., 2014, 46, 947961.

4 D. M. Qato, G. C. Alexander, R. M. Conti, M. Johnson, P. Schumm and S. T. Lindau, Use of prescription and overthe-counter medications and dietary supplements among older adults in the United States, J. Am. Med. Assoc., 2008, 300, 2867-2878.

5 O. Oyebode, V. Gordon-Dseagu, A. Walker and J. S. Mindell, Fruit and vegetable consumption and all-cause, cancer and CVD mortality: analysis of Health Survey for England data, J. Epidemiol. Community Health, 2014, 68, 856-862.
6 F. B. Hu, E. B. Rimm, M. J. Stampfer, A. Ascherio, D. Spiegelman and W. C. Willett, Prospective study of major dietary patterns and risk of coronary heart disease in men, Am. J. Clin. Nutr., 2000, 72, 912-921.

7 S. C. Chai, S. Hooshmand, R. L. Saadat, M. E. Payton, K. Brummel-Smith and B. H. Arjmandi, Daily apple versus dried plum: impact on cardiovascular disease risk factors in postmenopausal women, J. Acad. Nutr. Diet., 2012, 112, 1158-1168.

8 S. A. Johnson, A. Figueroa, N. Navaei, A. Wong, R. Kalfon, L. T. Ormsbee, R. G. Feresin, M. L. Elam, S. Hooshmand, M. E. Payton and B. H. Arjmandi, Daily blueberry consumption improves blood pressure and arterial stiffness in postmenopausal women with pre- and stage 1-hypertension: a randomized, double-blind, placebo-controlled clinical trial, J. Acad. Nutr. Diet., 2015, 115, 369-377.

9 A. Basu, M. Wilkinson, K. Penugonda, B. Simmons, N. M. Betts and T. J. Lyons, Freeze-dried strawberry powder improves lipid profile and lipid peroxidation in women with metabolic syndrome: baseline and post intervention effects, Nutr. J., 2009, 8, 43.

10 B. L. Halvorsen, M. H. Carlsen, K. M. Phillips, S. K. Bohn, K. Holte, D. R. Jacobs Jr. and R. Blomhoff, Content of redox-active compounds (ie, antioxidants) in foods consumed in the United States, Am. J. Clin. Nutr., 2006, 84, 95135.

11 T. Traustadottir, S. S. Davies, A. A. Stock, Y. Su, C. B. Heward, L. J. Roberts 2nd and S. M. Harman, Tart cherry juice decreases oxidative stress in healthy older men and women, J. Nutr., 2009, 139, 1896-1900.

12 B. Ou, K. N. Bosak, P. R. Brickner, D. G. Iezzoni and E. M. Seymour, Processed tart cherry products-comparative phytochemical content, in vitro antioxidant capacity and in vitro anti-inflammatory activity, J. Food Sci., 2012, 77, H105-H112.

13 V. Mulabagal, G. A. Lang, D. L. DeWitt, S. S. Dalavoy and M. G. Nair, Anthocyanin content, lipid peroxidation and cyclooxygenase enzyme inhibitory activities of sweet and sour cherries, J. Agric. Food Chem., 2009, 57, 1239-1246.

14 A. Saric, S. Sobocanec, T. Balog, B. Kusic, V. Sverko, V. Dragovic-Uzelac, B. Levaj, Z. Cosic, Z. Macak Safranko and T. Marotti, Improved antioxidant and anti-inflammatory potential in mice consuming sour cherry juice (Prunus Cerasus cv. Maraska), Plant Foods Hum. Nutr., 2009, 64, 231-237.

15 K. S. Kuehl, E. T. Perrier, D. L. Elliot and J. C. Chesnutt, Efficacy of tart cherry juice in reducing muscle pain during running: a randomized controlled trial, J. Int. Soc. Sports Nutr., 2010, 7, 17.

16 G. Howatson, P. G. Bell, J. Tallent, B. Middleton, M. P. McHugh and J. Ellis, Effect of tart cherry juice (Prunus cerasus) on melatonin levels and enhanced sleep quality, Eur. J. Nutr., 2012, 51, 909-916.

17 G. Howatson, M. P. McHugh, J. A. Hill, J. Brouner, A. P. Jewell, K. A. van Someren, R. E. Shave and S. A. Howatson, Influence of tart cherry juice on indices of 
recovery following marathon running, Scand. J. Med. Sci. Sports, 2010, 20, 843-852.

18 W. R. Pigeon, M. Carr, C. Gorman and M. L. Perlis, Effects of a tart cherry juice beverage on the sleep of older adults with insomnia: a pilot study, J. Med. Food, 2010, 13, 579583.

19 E. M. Seymour, S. K. Lewis, D. E. Urcuyo-Llanes, I. I. Tanone, A. Kirakosyan, P. B. Kaufman and S. F. Bolling, Regular tart cherry intake alters abdominal adiposity, adipose gene transcription, and inflammation in obesityprone rats fed a high fat diet, J. Med. Food, 2009, 12, 935942.

20 E. M. Seymour, A. A. Singer, A. Kirakosyan, D. E. UrcuyoLlanes, P. B. Kaufman and S. F. Bolling, Altered hyperlipidemia, hepatic steatosis, and hepatic peroxisome proliferator-activated receptors in rats with intake of tart cherry, J. Med. Food, 2008, 11, 252-259.

21 A. Ataie-Jafari, S. Hosseini, F. Karimi and M. Pajouhi, Effects of sour cherry juice on blood glucose ad some cardiovascular risk factors improvements in diabetic women: a pilot study, Nutr. Food Sci., 2008, 38, 355-360.

22 K. M. Keane, T. W. George, C. L. Constantinou, M. A. Brown, T. Clifford and G. Howatson, Effects of Montmorency tart cherry (Prunus Cerasus L.) consumption on vascular function in men with early hypertension, Am. J. Clin. Nutr., 2016, 103, 1531-1539.

23 W. T. Friedewald, R. I. Levy and D. S. Fredrickson, Estimation of the concentration of low-density lipoprotein cholesterol in plasma, without use of the preparative ultracentrifuge, Clin. Chem., 1972, 18, 499-502.

24 R. A. Washburn, E. McAuley, J. Katula, S. L. Mihalko and R. A. Boileau, The physical activity scale for the elderly (PASE): evidence for validity, J. Clin. Epidemiol., 1999, 52, 643-651.

25 A. Lynn, S. Mathew, C. T. Moore, J. Russell, E. Robinson, V. Soumpasi and M. E. Barker, Effect of a tart cherry juice supplement on arterial stiffness and inflammation in healthy adults: a randomised controlled trial, Plant Foods Hum. Nutr., 2014, 69, 122-127.

26 H. Wang, M. G. Nair, G. M. Strasburg, Y. C. Chang, A. M. Booren, J. I. Gray and D. L. DeWitt, Antioxidant and antiinflammatory activities of anthocyanins and their aglycon, cyanidin, from tart cherries, J. Nat. Prod., 1999, 62, 802 .

27 N. P. Seeram, L. D. Bourquin and M. G. Nair, Degradation products of cyanidin glycosides from tart cherries and their bioactivities, J. Agric. Food Chem., 2001, 49, 49244929.

28 M. Diebolt, B. Bucher and R. Andriantsitohaina, Wine polyphenols decrease blood pressure, improve NO vasodilatation, and induce gene expression, Hypertension, 2001, 38, 159-165.

29 I. Erlund, R. Koli, G. Alfthan, J. Marniemi, P. Puukka, P. Mustonen, P. Mattila and A. Jula, Favorable effects of berry consumption on platelet function, blood pressure, and HDL cholesterol, Am. J. Clin. Nutr., 2008, 87, 323-331.
30 J. P. Kamat, A. Ghosh and T. P. Devasagayam, Vanillin as an antioxidant in rat liver mitochondria: inhibition of protein oxidation and lipid peroxidation induced by photosensitization, Mol. Cell. Biochem., 2000, 209, 47-53.

31 S. Kakkar and S. Bais, A review on protocatechuic Acid and its pharmacological potential, ISRN Pharmacol., 2014, 2014, 952943.

32 K. M. Keane, P. G. Bell, J. K. Lodge, C. L. Constantinou, S. E. Jenkinson, R. Bass and G. Howatson, Phytochemical uptake following human consumption of Montmorency tart cherry (L. Prunus cerasus) and influence of phenolic acids on vascular smooth muscle cells in vitro, Eur. J. Nutr., 2016, 55, 1695-1705.

33 D. A. Hobbs, N. Kaffa, T. W. George, L. Methven and J. A. Lovegrove, Blood pressure-lowering effects of beetroot juice and novel beetroot-enriched bread products in normotensive male subjects, Br. J. Nutr., 2012, 108, 20662074.

34 P. K. Whelton, J. He, J. A. Cutler, F. L. Brancati, L. J. Appel, D. Follmann and M. J. Klag, Effects of oral potassium on blood pressure. Meta-analysis of randomized controlled clinical trials, J. Am. Med. Assoc., 1997, 277, 1624-1632.

35 J. M. Geleijnse, F. J. Kok and D. E. Grobbee, Blood pressure response to changes in sodium and potassium intake: a metaregression analysis of randomised trials, J. Hum. Hypertens., 2003, 17, 471-480.

36 K. T. Khaw and E. Barrett-Connor, Dietary potassium and stroke-associated mortality. A 12-year prospective population study, N. Engl. J. Med., 1987, 316, 235-240.

37 K. T. Khaw and G. Rose, Population study of blood pressure and associated factors in St Lucia, West Indies, Int. J. Epidemiol., 1982, 11, 372-377.

38 L. J. Appel, T. J. Moore, E. Obarzanek, W. M. Vollmer, L. P. Svetkey, F. M. Sacks, G. A. Bray, T. M. Vogt, J. A. Cutler, M. M. Windhauser, P. H. Lin and N. Karanja, A clinical trial of the effects of dietary patterns on blood pressure. DASH Collaborative Research Group, N. Engl. J. Med., 1997, 336, 1117-1124.

39 N. J. Aburto, S. Hanson, H. Gutierrez, L. Hooper, P. Elliott and F. P. Cappuccio, Effect of increased potassium intake on cardiovascular risk factors and disease: systematic review and meta-analyses, Br. Med. J., 2013, 346, f1378.

40 A. Sagie, M. G. Larson and D. Levy, The natural history of borderline isolated systolic hypertension, N. Engl. J. Med., 1993, 329, 1912-1917.

41 R. Stamler, Implications of the INTERSALT study, Hypertension, 1991, 17, I16-I20.

42 P. Barter, A. M. Gotto, J. C. LaRosa, J. Maroni, M. Szarek, S. M. Grundy, J. J. Kastelein, V. Bittner, J. C. Fruchart and I. Treating to New Targets, HDL cholesterol, very low levels of LDL cholesterol, and cardiovascular events, N. Engl. J. Med., 2007, 357, 1301-1310.

43 S. H. Hsia, D. Pan, P. Berookim and M. L. Lee, A population-based, cross-sectional comparison of lipid-related indexes for symptoms of atherosclerotic disease, Am. J. Cardiol., 2006, 98, 1047-1052. 
44 T. S. Kahlon and G. E. Smith, In vitro binding of bile acids by blueberries (Vaccinium spp.), plums (Prunus spp.), strawberries (Fragaria X ananassa), cherries (Malpighia punicifolia), cranberries (Vaccinium macrocarpon) and apples (Malus sylvestris), Food Chem., 2007, 100, 11821187.

45 I. M. Heinonen, P. J. Lehtonen and A. I. Hopia, Antioxidant Activity of Berry and Fruit Wines and Liquors, J. Agric. Food Chem., 1998, 46, 25-31.

46 F. Jameel, M. Phang, L. G. Wood and M. L. Garg, Acute effects of feeding fructose, glucose and sucrose on blood lipid levels and systemic inflammation, Lipids Health Dis., 2014, 13, 195.

47 C. M. Brown, A. G. Dulloo, G. Yepuri and J. P. Montani, Fructose ingestion acutely elevates blood pressure in healthy young humans, Am. J. Physiol.: Regul., Integr. Comp. Physiol., 2008, 294, R730-R737.
48 M. C. Litterio, M. A. Vazquez Prieto, A. M. Adamo, R. Elesgaray, P. I. Oteiza, M. Galleano and C. G. Fraga, (-)-Epicatechin reduces blood pressure increase in highfructose-fed rats: effects on the determinants of nitric oxide bioavailability, J. Nutr. Biochem., 2015, 26, 745-751.

49 K. L. Stanhope, J. M. Schwarz, N. L. Keim, S. C. Griffen, A. A. Bremer, J. L. Graham, B. Hatcher, C. L. Cox, A. Dyachenko, W. Zhang, J. P. McGahan, A. Seibert, R. M. Krauss, S. Chiu, E. J. Schaefer, M. Ai, S. Otokozawa, K. Nakajima, T. Nakano, C. Beysen, M. K. Hellerstein, L. Berglund and P. J. Havel, Consuming fructose-sweetened, not glucose-sweetened, beverages increases visceral adiposity and lipids and decreases insulin sensitivity in overweight/obese humans, J. Clin. Invest., 2009, 119, 1322-1334.

50 E. Han and L. M. Powell, Consumption patterns of sugarsweetened beverages in the United States, J. Acad. Nutr. Diet., 2013, 113, 43-53. 